\title{
Inhomogeneities on the surface of 21 Lutetia, the asteroid target of the Rosetta mission
}

\section{Ground-based results before the Rosetta fly-by}

\author{
D. Perna ${ }^{1,2,3}$, E. Dotto ${ }^{3}$, M. Lazzarin ${ }^{4}$, S. Magrin ${ }^{4}$, M. Fulchignoni ${ }^{2,5}$, M. A. Barucci ${ }^{2}$, S. Fornasier ${ }^{2,5}$, \\ S. Marchi ${ }^{4}$, and C. Barbieri ${ }^{4}$ \\ 1 Dipartimento di Fisica, Università di Roma Tor Vergata, via della Ricerca Scientifica 1, 00133 Roma, Italy \\ e-mail: dperna@oa-roma.inaf.it \\ 2 LESIA, Observatoire de Paris, 5 Place J. Janssen, 92195 Meudon Principal Cedex, France \\ 3 INAF - Osservatorio Astronomico di Roma, via Frascati 33, 00040 Monte Porzio Catone (Roma), Italy \\ 4 Dipartimento di Astronomia, Università di Padova, Vicolo dell'Osservatorio 3, 35122 Padova, Italy \\ 5 Université Paris Diderot - Paris 7, 4 rue Elsa Morante, 75013 Paris, France
}

Received 12 January 2010 / Accepted 7 March 2010

\section{ABSTRACT}

\begin{abstract}
Context. In July 2010 the ESA spacecraft Rosetta will fly by the main belt asteroid 21 Lutetia. Several observations of this asteroid have been performed so far, but its surface composition and nature are still a matter of debate. For a long time Lutetia was supposed to have a metallic nature due to its high IRAS albedo. Later on it has been suggested that the asteroid has a surface composition similar to primitive carbonaceous chondrite meteorites, while further observations proposed a possible genetic link with more evolved enstatite chondrite meteorites.

Aims. We performed visible spectroscopic observations of 21 Lutetia in November 2008 at the Telescopio Nazionale Galileo (TNG, La Palma, Spain) to make a decisive contribution to solving the conundrum of its nature.

Methods. Thirteen visible spectra were acquired at different rotational phases and subsequently analyzed.

Results. We confirm a narrow spectral feature at about $0.47-0.48 \mu \mathrm{m}$ which was already found by Lazzarin et al. (2004, A\&A, 425, L25) in the spectra of Lutetia. We also confirm an earlier find of Lazzarin et al. (2004), who detected a spectral feature at about $0.6 \mu \mathrm{m}$ in one of their Lutetia's spectra. More remarkable is the difference of our spectra though, which exhibit different spectral slopes between 0.6 and $0.75 \mu \mathrm{m}$ and, in particular, we found that up to $20 \%$ of the Lutetia surface could have flatter spectra. Conclusions. We detected a variation of the spectral slopes at different rotational phases that could be interpreted as possibly due to differences in the chemical/mineralogical composition as well as to inhomogeneities of the structure of the Lutetia's surface (e.g., to craters or albedo spots) in the southern hemisphere.
\end{abstract}

Key words. techniques: spectroscopic - minor planets, asteroids: individual: Lutetia

\section{Introduction}

Rosetta is the ESA cornerstone mission devoted to the study of minor bodies of the solar system. The main target is comet 67P/Churyumov-Gerasimenko that will be reached in 2014, after three Earth and one Mars gravity assisted swing-bys. During its journey the mission investigates also two main belt asteroids, 2867 Steins (fly-by in September 2008) and 21 Lutetia (fly-by in July 2010).

The asteroid 21 Lutetia was discovered in 1852 by Goldschmidt at the Paris Observatory. Zappalà et al. (1984) measured a rotational period of $P=8.17 \pm 0.01 \mathrm{~h}$, a value later refined by Torppa et al. (2003), who found $P=8.165455 \mathrm{~h}$ and also computed the pole coordinates obtaining a model with axis ratios $a / b=1.2$ and $b / c=1.2$. Although several

* Based on observations made with the Italian Telescopio Nazionale Galileo (TNG) operated on the island of La Palma by the Fundacion Galileo Galilei of the INAF (Istituto Nazionale di Astrofisica) at the Spanish Observatorio del Roque de los Muchachos of the Instituto de Astrofisica de Canarias (DDT program partially performed during program AOT18/TAC21). observations are available since a couple of decades, the nature of this asteroid is still controversial. Radiometric measurements gave albedo values included in the range 0.19-0.22 (IRAS, Müller et al. 2006; Lamy et al. 2008). More recently, Carvano et al. (2008) obtained a geometric albedo of 0.129, significantly lower than all the previous estimations, and explained the wide range of computed albedo values as the evidence of inhomogeneities on the surface of Lutetia (e.g., one or more large craters on the northern hemisphere). Due to the first estimation of its albedo by IRAS, Lutetia was supposed to have a metallic nature (Barucci et al. 1987; Tholen 1989). Later on it has been suggested that the asteroid has a surface composition similar to primitive carbonaceous chondrite meteorites (Howell et al. 1994; Burbine \& Binzel 2002; Lazzarin et al. 2004, 2009; Birlan et al. 2004; Barucci et al. 2005, 2008), while Vernazza et al. (2009) proposed a possible genetic link with more evolved enstatite chondrite meteorites.

On the basis of the observational evidence, Lutetia appears to be an atypical puzzling asteroid, whose nature is still far from fully understood. In order to enhance our knowledge of this unusual object, in November 2008 we performed visible 
Table 1. Observational circumstances.

\begin{tabular}{cccccccc}
\hline \hline$\#$ & Date & $U T_{\text {start }}$ & $T_{\exp }$ & Airm. & $\begin{array}{c}\text { Spec. } \\
\text { slope } \\
\left(\% / 10^{3} \AA\right)\end{array}$ & $\begin{array}{c}\text { Rot. } \\
\text { phase }\end{array}$ & $\begin{array}{c}\text { Sol. an. } \\
\text { airm. }\end{array}$ \\
& & & $(\mathrm{hh}: \mathrm{mm})$ & $(\mathrm{s})$ & & \\
\hline 1 & 2008 Nov. 27 & $23: 39$ & 8 & 1.07 & $1.5 \pm 0.5$ & 0.018 & 1.04 \\
2 & 2008 Nov. 27 & $23: 53$ & 30 & 1.05 & $1.0 \pm 0.5$ & 0.046 & 1.04 \\
3 & 2008 Nov. 28 & $00: 49$ & 40 & 1.01 & $1.4 \pm 0.5$ & 0.161 & 1.04 \\
4 & 2008 Nov. 28 & $01: 33$ & 40 & 1.02 & $1.8 \pm 0.5$ & 0.251 & 1.04 \\
5 & 2008 Nov. 28 & $02: 23$ & 40 & 1.07 & $2.2 \pm 0.5$ & 0.353 & 1.04 \\
6 & 2008 Nov. 28 & $03: 05$ & 40 & 1.14 & $2.0 \pm 0.5$ & 0.438 & 1.04 \\
7 & 2008 Nov. 28 & $03: 54$ & 40 & 1.30 & $2.1 \pm 0.5$ & 0.538 & 1.04 \\
8 & 2008 Nov. 28 & $04: 32$ & 40 & 1.50 & $1.7 \pm 0.5$ & 0.616 & 1.04 \\
9 & 2008 Nov. 29 & $22: 18$ & 40 & 1.23 & $0.7 \pm 0.5$ & 0.731 & 1.02 \\
10 & 2008 Nov. 29 & $23: 12$ & 40 & 1.09 & $1.4 \pm 0.5$ & 0.841 & 1.02 \\
11 & 2008 Nov. 29 & $23: 13$ & 30 & 1.09 & $1.6 \pm 0.5$ & 0.843 & 1.02 \\
12 & 2008 Nov. 30 & $00: 07$ & 30 & 1.03 & $1.7 \pm 0.5$ & 0.953 & 1.02 \\
13 & 2008 Nov. 30 & $00: 57$ & 40 & 1.01 & $1.7 \pm 0.5$ & 0.056 & 1.02 \\
\hline
\end{tabular}

Notes. For each spectrum we report the starting time of acquisition corrected for light-time, the exposure length, the airmass of Lutetia, the spectral slope computed between $0.6 \mu \mathrm{m}$ and $0.75 \mu \mathrm{m}$, the coverage of the rotational phase, and the airmass of the used solar analog.

spectroscopic observations of Lutetia to investigate its surface and check if some inhomogeneities are present.

\section{Observations and data reduction}

Observations were carried out at the $3.6 \mathrm{~m}$ Telescopio Nazionale Galileo (TNG, La Palma, Spain). The observational circumstances are given in Table 1.

Visible spectroscopy was performed with the DOLORES (Device Optimized for the LOw RESolution) instrument. We used the low resolution blue (LR-B) grism, which cover the $0.34-0.81 \mu \mathrm{m}$ range with a spectral dispersion of $2.5 \AA / \mathrm{px}$ (http://www.tng.iac.es). Spectra were taken through a 2 arcsec wide slit oriented along the parallactic angle to avoid flux loss due to the differential refraction. Bias, flat-field, calibration lamp (He lines) and several spectra of solar analog stars (Landolt 98-978 and Hyades 64) were recorded during the observing run. The spectra were reduced with the software packages Midas and IDL using standard procedures (see, e.g., Dotto et al. 2009) which include subtraction of the bias from the raw data, flat field correction, cosmic rays removal, background subtraction, collapsing the two-dimensional spectra into one dimension, wavelength calibration (using the He lamp's emission lines) and atmospheric extinction correction. The reflectivity of Lutetia was then obtained by dividing its spectra by one of the solar analog star Hyades 64. The resulting spectra were cut at $0.45 \mu \mathrm{m}$ because at lower wavelengths the behavior is strongly affected by the spectra of the solar analogs. Table 1 also reports the coverage of the rotational phase of each of the obtained spectra and the spectral slopes computed between $0.6 \mu \mathrm{m}$ and $0.75 \mu \mathrm{m}$.

\section{Obtained results and discussion}

The obtained spectra are shown in Fig. 1. The general behavior is similar in all of them, but some differences are evident.

All spectra exhibit two weak features at about $0.47-0.48 \mu \mathrm{m}$ and around $0.6 \mu \mathrm{m}$. Their depth relative to the continuum reflectance is of about $1 \%$ in all spectra, and does not exhibit any evident variation. Bus \& Binzel (2002a,b) observed features at

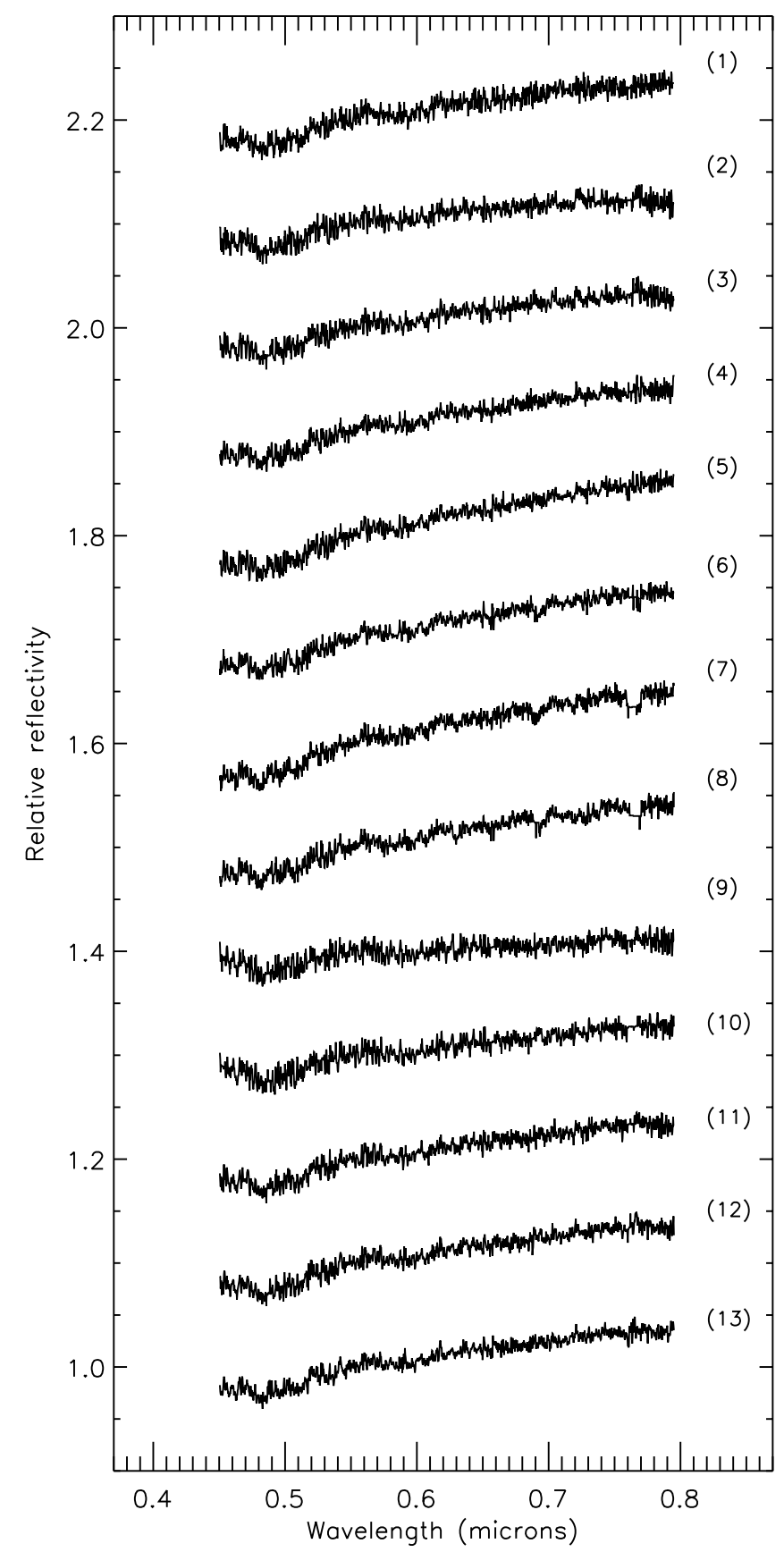

Fig. 1. Visible spectra of Lutetia normalized at $0.55 \mu \mathrm{m}$ and shifted by 0.1 in reflectivity for clarity. The numbers on the right correspond to the spectrum number reported in Table 1 and Fig. 2.

about $0.48 \mu \mathrm{m}$ and $0.6 \mu \mathrm{m}$ in the spectra of several main belt asteroids, which were therefore classified as Xe types. Burbine et al. (2002) found that these spectral signatures are consistent with those detected in oldhamite, a mineral commonly found in the aubrites (enstatite achondrite meteorites), but which is also present in enstatite chondrites. Vernazza et al. (2009), who analyzed visible spectra and discussed the computed albedo values, suggested that the physical properties of Lutetia are compatible with those of enstatite chondrite meteorites. Nevertheless, the shape of the feature at about $0.48 \mu \mathrm{m}$ in the Lutetia spectra is considerably different from that observable in Xe-type asteroids, probably indicating a dissimilar origin of the absorption. 
D. Perna et al.: 21 Lutetia: Ground-based results before the Rosetta fly-by

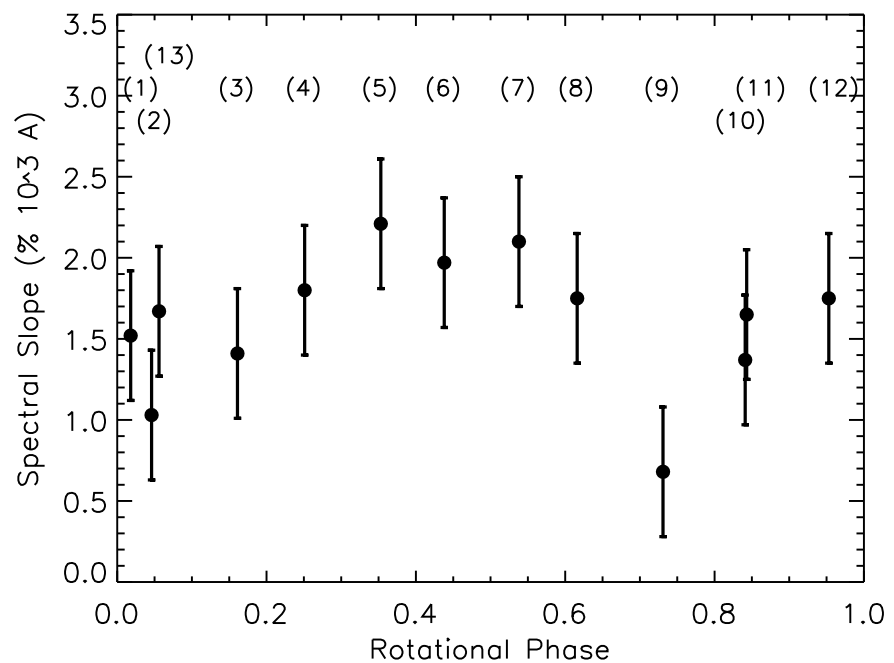

Fig. 2. Spectral slope vs. rotational phase, computed considering a period of $8.165455 \mathrm{~h}$ (Torppa et al. 2003).

Both features at $\sim 0.48 \mu \mathrm{m}$ and $0.6 \mu \mathrm{m}$ were already detected in the spectra of Lutetia (Lazzarin et al. 2004, 2009). The signature at about $0.47-0.48 \mu \mathrm{m}$ was detected by Lazzarin et al. (2009) and interpreted as due to spin-allowed crystal field transitions in $\mathrm{Ti}^{3+}$ in pyroxene M1 sites, although Hazen et al. (1978) suggested that in lunar pyroxenes this band could be due to the superimposition of $\mathrm{Ti}^{3+}$ and $\mathrm{Fe}^{2+}$ effects. The feature at about $0.6 \mu \mathrm{m}$ was seen in one of the spectra published by Lazzarin et al. (2004) and it is generally attributed to charge transfer transitions in minerals produced by aqueous alteration of anhydrous silicates (Vilas et al. 1994), but can be also present in pyroxenes (see, e.g., Burns et al. 1976). Additional constraints come from far-infrared Spitzer data of Lutetia, which do not confirm any similarity with enstatite chondrite meteorites (Barucci et al. 2008; Lazzarin et al. 2009), but suggest CO3-CV3 carbonaceous chondrites as the closest meteorite analogs. Because the mineralogical composition of asteroids can be inferred only by taking into account multiwavelength observational data together with albedo values, the most plausible interpretation of the features detected in visible spectra of Lutetia at this point in time appears to be that given by Lazzarin et al. (2004, 2009), while the analogy with enstatite chondrites seems unlikely.

Figure 2 plots the obtained spectral slopes (values reported in Table 1). A variation through the rotational phase is evident and can be interpreted as due to inhomogeneities on the observed portion of the Lutetia's surface. These inhomogeneities could be related to different chemical/mineralogical compositions, as well as to albedo spots or to craters exposing regions with different albedo/age. In particular, a clear inhomogeneity emerges in correspondence with spectrum \# 9 , which could cover up to $20 \%$ of the surface we observed.
Considering the pole solution $\left(\lambda=39^{\circ}, \beta=3^{\circ}\right)$ by Torppa et al. (2003), our spectra were obtained at an aspect angle $\xi \sim 30^{\circ}$. Hence we can support the hypothesis that one or more craters/inhomogeneities can be present in the southern hemisphere of Lutetia.

\section{Conclusions}

An observational campaign of Lutetia was carried out on November 2008. Visible spectroscopy was performed for this main belt asteroid and thirteen spectra were acquired at different rotational phases. All of them exhibit absorption features centered at about $0.47-0.48 \mu \mathrm{m}$ and around $0.6 \mu \mathrm{m}$. The spectral slope shows a variation through the rotational phase, suggesting that some inhomogeneities must be present in a portion of up to $20 \%$ of the observed surface of Lutetia in its southern hemisphere. These differences in the acquired spectra can be due to inhomogeneities in the chemical/mineralogical composition, or to albedo spots or craters exposing regions with different albedo/age.

These data are useful in the assessment of the physical nature of this object, and will constitute a fundamental basis for the calibration, analysis and interpretation of the data acquired by the instruments onboard the Rosetta spacecraft.

Acknowledgements. We thank Emilio Molinari for the DDT shared between the groups of E. Dotto and M. Lazzarin. We are grateful to Francesca Ghinassi for help and useful discussions during the observations. We also thank T.H. Burbine, referee of the manuscript, for providing constructive comments and help in improving this paper.

\section{References}

Barucci, M. A., Capria, M. T., Coradini A., et al. 1987, Icarus, 72, 304 Barucci, M. A., Fulchignoni, M., Fornasier, S., et al. 2005, A\&A, 430, 313 Barucci, M. A., Fornasier, S., Dotto, E., et al. 2008, A\&A, 477, 665 Birlan, M., Barucci, M. A., Vernazza, P., et al. 2004, New Astron., 9, 343 Burbine, T. H., \& Binzel, R. P. 2002, Icarus, 159, 468

Burbine, T. H., McCoy, T. J., Nittler, L. R., et al. 2002, Meteoritics \& Planetary Science, 37, 1233

Burns, R. G., Parkin, K. M., Loeffler, B. M., et al. 1976, LPSC, 7, 2561

Bus, S. J., \& Binzel, R. P. 2002a, Icarus, 158, 106

Bus, S. J., \& Binzel, R. P. 2002b, Icarus, 158, 146

Carvano, J. M., Barucci, M. A., Delbó, M., et al. 2008, A\&A, 479, 241

Dotto, E., Perna, D., Fornasier, S., et al. 2009, A\&A, 494, L29

Hazen, R. M., Bell, P. M., \& Mao, H. K. 1978, LPSC, 9, 2919

Howell, E. S., Merenyi, E., \& Lebofsky, L. A. 1994, JGR, 99, 10848

Lamy, P. L., Barucci, M. A., Fornasier, S., et al. 2008, ACM 2008 abstract, 8245 Lazzarin, M., Marchi, S., Magrin, S., et al. 2004, A\&A, 425, L25

Lazzarin, M., Marchi, S., Moroz, L. V., et al. 2009, A\&A, 498, 307

Müller, M., Harris, A. W., Bus, S. J., et al. 2006, A\&A, 447, 1153

Tholen, D. 1989, in Asteroids II, ed. R. P. Binzel, T. Gehrels, \& M. S. Matthews (Univ. of Arizona Press, Tucson), 1139

Torppa, J., Kaasalainen, M., Michalowski, T., et al. 2003, Icarus, 164, 346

Vilas, F., Jarvis, K. S., \& Gaffey, M. J. 1994, Icarus, 109, 274

Vernazza, P., Brunetto, R., Binzel, R. P., et al. 2009, Icarus, 202, 477

Zappalà, V., Di Martino, M., Knezevic, Z., et al. 1984, A\&A, 130, 208 